\title{
COMPARATIVE MORPHOLOGICAL ANALYSIS OF ENAMEL AND DENTIN SURFACES' RECONSTRUCTIONS BY MEANS OF AUTOMATED DIGITAL ODONTOMETRY
}

\author{
A.V. Gaboutchian ${ }^{1 *}$, V. A. Knyaz ${ }^{2,3}$, M.M. Novikov ${ }^{4}$, S.V. Vazyliev ${ }^{5}$, D.V. Korost ${ }^{6}$, S.A. Cherebylo ${ }^{4}$, A.A. Kudaev $^{6}$ \\ ${ }^{1}$ Peoples Friendship University of Russia, 117198, Moscow, Russia armengaboutchian@ mail.ru \\ ${ }^{2}$ State Research Institute of Aviation System (GosNIIAS), 125319 Moscow, Russia - knyaz@gosniias.ru \\ ${ }^{3}$ Moscow Institute of Physics and Technology (MIPT), Dolgoprudny, Russia \\ ${ }^{4}$ Research Center Crystallography and Photonics RAS, Shatura, Russia - novikov@ rambler.ru \\ ${ }^{5}$ Institute of Ethnology and Anthropology RAS, Moscow, Russia - vasbor1@ yandex.ru \\ ${ }^{6}$ Faculty of Geology, Moscow State University, 119234, Moscow, Russia dkorost@mail.ru ; a.a.kudaev@gmail.com
}

\section{Commission II, WG II/10}

KEY WORDS: automated digital odontometry, micro-computed tomography, dental morphology, measurements of dentin, Sunghir

\begin{abstract}
:
Studies of teeth represent a significant part of palaeoanthropological research. Over the past two decades these studies have significantly developed with implementation of high resolution imaging based on x-ray scanning techniques. Highly informative reconstructions based on image processing have provided an opportunity to study morphological layers and structures of teeth which are usually hidden under the outer layer of dental enamel. Thus micro-computed tomography of the studied teeth has been performed in order to obtain reconstructions of enamel and dentin surfaces. The material is represented by well-preserved teeth of an adolescent from Upper Palaeolithic archaeological site of Sunghir world-renowned archaeological site in Vladimir Oblast in the Russian Federation. The characteristic feature of the studied teeth is in their unusual, presumably archaic, morphology, which has been previously studied and described through measurements by application of automated digital odontometry method; however the mentioned study referred to the enamel surface. And in the current study these algorithms are applied to measure the surface of dentin. As this is the first successful attempt of measuring dentin surface morphology, the process has to be improved for complete automation. Nevertheless even currently applied approaches allow to compare enamel and dentin morphology through measurements.
\end{abstract}

\section{INTRODUCTION}

Studies of internal structures of teeth, their morphology and histology, have been traditionally carried out in palaeoanthropology and palaeontology. The most wide-spread way of obtaining information has been sectioning teeth. And it still remains, if we talk about histological research. However, being a highly informative study method, its main disadvantage refers to the fact that it is a highly invasive approach inevitably destroying findings; and the vast majority of such findings are unique. Another approach to collecting data about inner structures is conventional x-ray imaging; however planar imaging of volumetric objects can be more likely suitable for qualitative assessments.

\section{RELATED WORK}

Significantly greater potential for such studies have been proposed with relatively recent introduction of micro-computed tomography scanning and imaging techniques. It has provided high resolution reconstructions of inner and outer dental morphology including the complete layer of dental enamel, dentin and pulpal chamber. This has increased potential opportunities for dental studies based on visual analysis of morphology. This is due to high resolution $3 \mathrm{~d}$ imaging which made visible important morphological details of deeper layers of dental structure, previously unavailable for researchers in full geometric volume even after

${ }^{*}$ Corresponding author sectioning. Thus internal, located on the surface of dentin, morphological characteristics can be described and assessed in manner similar to odontological (or dental anthropological, depending on terminological traditions) evaluation of enamel surface (Bermdez de Castro et al., 2017). In line with visual studies a new impetus receives geometric morphometric research. Thus shape analysis, previously applied exceptionally to studies of external surface of dental enamel (Robinson et al., 2002), has been successfully applied to studies of dentin surface. Moreover, comparatively small morphological details can be studied using geometric morphometric analysis (Ortiz et al., 2019). However today high resolution reconstructions obtained through microcomputed tomography are widely used in studies of external and internal morphological structures. Such studies cover a wide range of territories and historical periods and, related to them, anthropological variety of extinct and living species (Hublin et al., 2017; Hershkovitz et al., 2018; Krenn et al., 2019).

Internal structures become a part of a significant, in terms of its applicability for taxonomical interpretations, research direction dealing with enamel thickness measurements. These studies have also received a new impetus with improvements in imaging and image processing techniques. Enamel thickness measurements and assessments are carried out in paleontological and palaeoanthropological studies, and methodological approaches to measuring process are widely discussed (Benazzi et al., 2014 b; Smith et al., 2018). High resolution reconstructions are a good alternative for traditional sectioning performed for measuring thickness 
of enamel layer. They reconstruct morphology essential for such assessments accurately. In line with that $3 \mathrm{~d}$ techniques provide freedom of choice in orientating sectioning planes, number of attempts is unlimited and, which is very important, these methods are not invasive and contribute to preserving findings. Of course, histological studies would still require obtaining slices, but they occupy a lesser segment of anthropological and paleontological studies, thus a significant number of findings can remain uncut. There are still a number of studies and measurements carried out on conventional radiographs (Nadendla et al., 2016; Machado et al., 2020). Nevertheless $3 \mathrm{~d}$ imaging and reconstruction techniques significantly increase variety of studied aspects as well as accuracy in comparison to traditional techniques.

We should also mention that modern imaging and processing methods do not prevent implementation of traditional techniques on the obtained reconstructions. Thus there is a significant number of studies successfully combining $3 \mathrm{~d}$ imaging and related to them research methods with traditional techniques. As an example classical measurements in mesio-distal and bucco-lingual directions are performed and fit together with micro-computed studies of external and internal dental morphology (Benazzi et al., 2014 a; Xing et al., 2014). Nevertheless the potential of precise $3 \mathrm{~d}$ reconstructions is not only in revealing previously unavailable for research structures in a new quality but also in the potential of methodological improvements. Thus we would like to present in the current paper what are the new opportunities for studies on the example of automated digital odontometry application on $3 \mathrm{~d}$ reconstructions.

The mentioned above and currently used method of automated digital odontometry has been applied to a number of previously conducted studies. They have shown that the suggested methodology including automated orientating and measuring algorithms is applicable to morphological research which has been carried out in dentistry and anthropology. Such studies have been performed on digital reconstructions obtained through precise microcomputed tomographic scanning and reconstructive techniques (Gaboutchian et al., 2020). Whilst the development of automated digital odontometry has been performed on digital reconstructions obtained through optical scanning, which has a potential to provide only reconstruction of external surfaces, i.e. dental enamel on tooth crowns and outer surfaces of roots. Implementation of micro-CT scanning techniques in these studies provides new prospects for research, however requires certain methodological improvements. Both aspects are presented in this paper as follows. The obtained information allows to reveal correlation of data obtained through measurements conducted on enamel and dentin. However the automated measurements performed on reconstructed dentin surfaces are a novelty approach in palaeoanthropological studies, where studies of dentin have a growing potential for further development (Yi et al., 2020).

\section{METHOD}

The first tooth measured through automated digital odontometry on both enamel (external) and dentin (internal) surfaces is the upper left second molar of C2 individual from Sunghir. It is a well-known Upper Palaeolithic archaeological site situated near the capital of Vladimir Oblast in the Russian Federation. Excavations have started there in late 50-s of the last century by O.N. Bader. Individual C2 is the elder of two adolescents found lying head-to-head in burial 2. Its denture has been earlier studied and described as possessing archaic features (Zubov, 2000). The findings dating estimates vary and cover a period between 25-35 thousand years. Nevertheless the remains are well-preserved and the complete upper dental arch is a whole with the skull. The external enamel surface on the tooth which currently studied (as well as its antimere) has been measured through automated digital odontometry. That work has revealed applicability of the used method for assessments of dental morphology even when tooth surfaces possess unusual features (Gaboutchian et al., 2020).

The scanning procedure was performed on GE phoenix $v \mid$ tome $\mid x$ $\mathrm{m}$ instrument suitable for work with skull-size objects. Imaging procedure resulted in obtaining .bmp format and 8-bit colour depth file stacks. The skull was orientated in the scanning camera to obtain the highest resolutions in the upper molar region (teeth presented in the current study). Thus approximately five hundred slices with $43 \mu \mathrm{m}$ inter-slice distance correspond to the tooth studied. These images were extracted from the entire image stack and processed as a separate stack. For $3 \mathrm{~d}$ image reconstruction Avizo 9.01 software was used. For segmentation of dental tis- sues according to their radiopacity Threshold tool was used, as well as Blow tool for cleaning out areas with similar radiopacity though not related to the object itself. Unconstructed smoothing was used during 3D model generation within allocated areas within image stacks. Subsequently data compression was performed in order to decrease the volume of files which were then exported in .stl and .x formats. Measurements were conducted on coronal parts of the tooth. This means that the dentin model was trimmed and the edge of enamel served as a borderline to separate root and coronal portions of reconstructed dentin (Figure 1b).

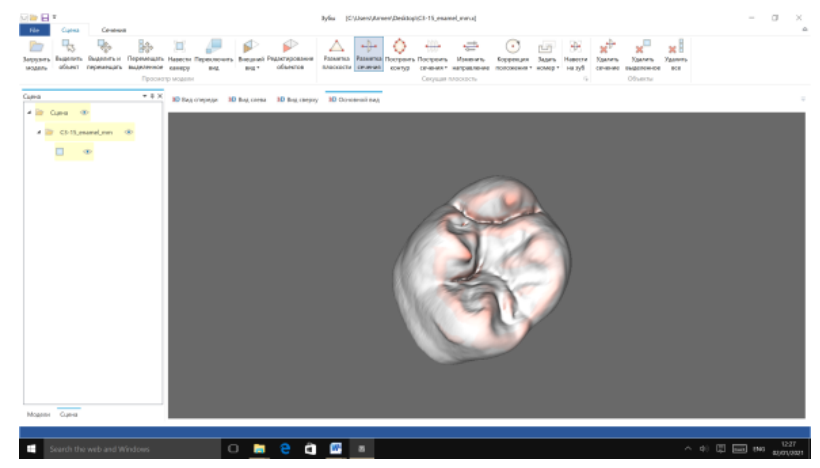

(a)

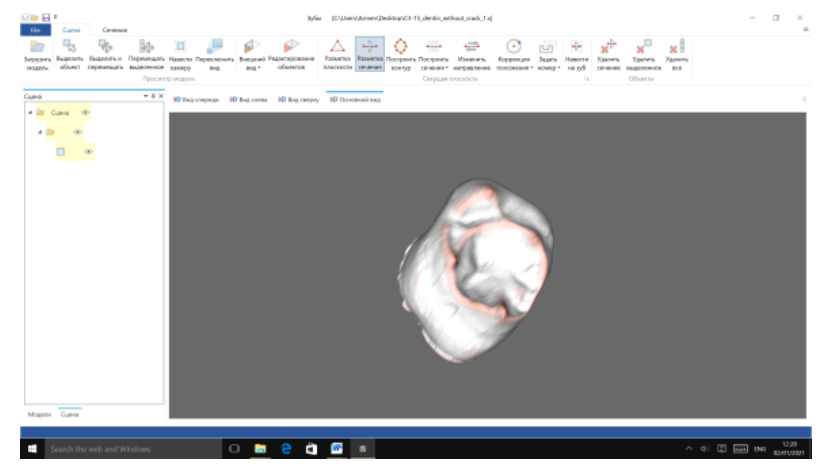

(b)

Figure 1. 3d reconstructions of enamel (a) and dentin (b) surfaces.

Subsequently the process of measurements passed through orientation stage. The standard approach to tooth orientation in the majority of palaeoanthropological studies requires an initial plane setting (Olejniczak et al., 2007; Zanolli et al., 2017; Benazzi et 
al., 2014 b). As an example, cervical plane can be set (usually in manual mode) and its inclination is approximated to the contour of the enamel edge. Another methodological approach suggests three dentine horn tips as landmarks for setting of plane orientation. Regarding the current study, approaches to orientation of teeth have been developed on the basis of $3 \mathrm{~d}$ surface analysis, especially referring to its curvature. They include fully automated algorithms of detection the morphological structure of anatomical occlusal surface borderline on the external enamel surface reconstructions; radial sectioning is applied as well. We should mention that the external surface of dentin corresponds to the enamel internal surfaces, though inner and outer surfaces of enamel are different. And this difference of reliefs does not allow direct implementation of the above-mentioned and successfully implemented algorithms for enamel surface to the surface of dentin; methodological improvements are required. So at the current stage of software development few manual adjustments were necessary to correct automatically set contour of the occlusal surface on dentin external surface reconstructions. These algorithms allow setting of axes and according to their orientation further algorithms are launched (Figure 2a,b). And the following step in automated digital odontometry is transverse sectioning of tooth crown.

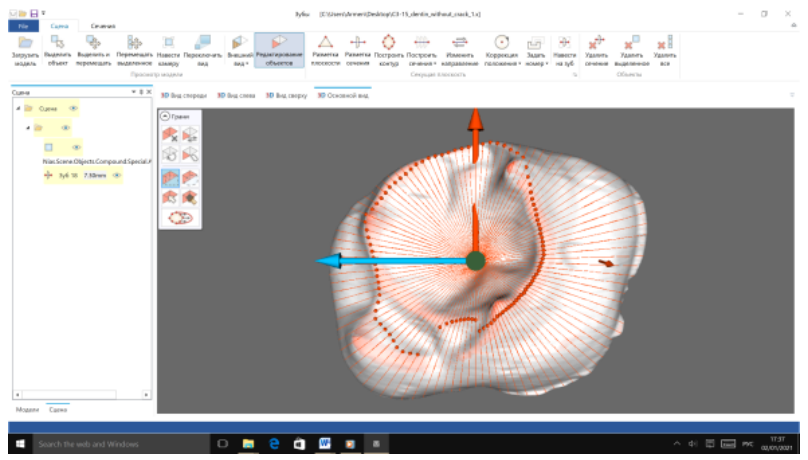

(a)

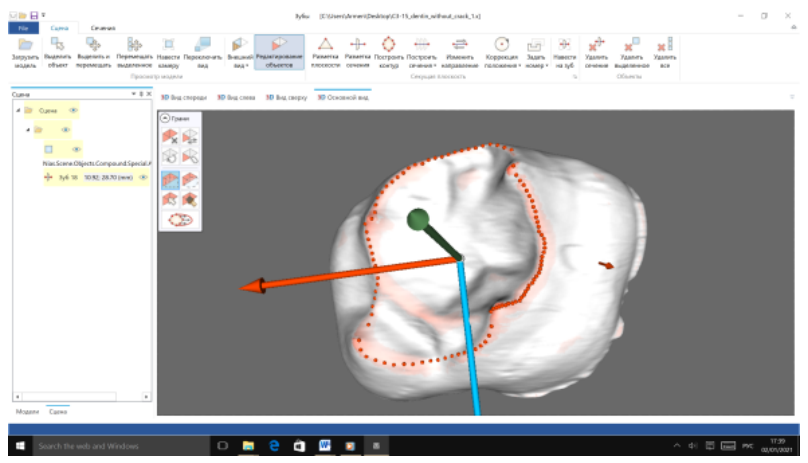

(b)

Figure 2. Orientation algorithms before (a) and after (b) manual adjustments.

As we have mentioned before sectioning is a significant part of many dental study methods as well as imaging techniques. Sections are also applied in automated digital odontometry for contour obtaining. And thus further contour obtaining algorithms are similar for enamel and dentin.

They perform a previously set number of parallel sections with equal intervals between slices evenly crossing the whole coronal part of the tooth in bucco-lingual (vestibular-oral) direction. It is set through preceding orientational algorithm perpendicular to the longitudinal axis of the tooth crown (Figure 3).

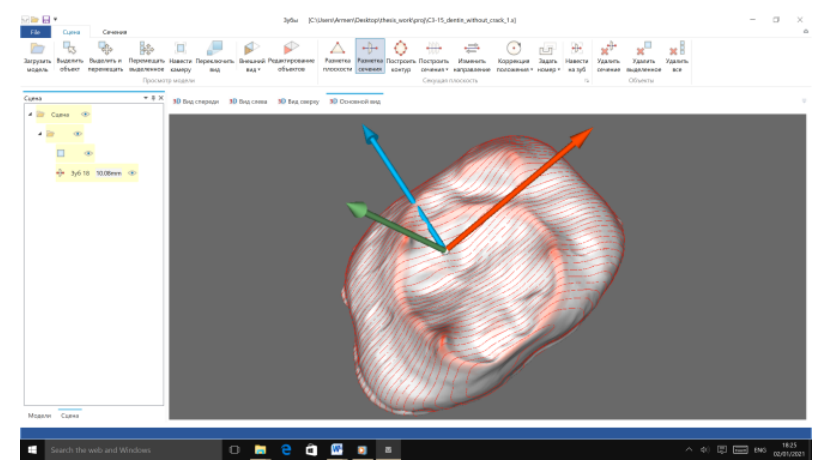

Figure 3. 40-slice sectioning on dentin reconstruction; the blue arrow indicates the longitudinal axis.

Measuring algorithms on dentin in the current study do not differ essentially from those on enamel. They are based on the same morphological interpretations and require similar set of landmarks on contour for corresponding geometric constructions and obtaining parameters. A wide variety of parameters: linear, angular, contour and coefficients, are obtained on each section (Figure $4 a, b)$.

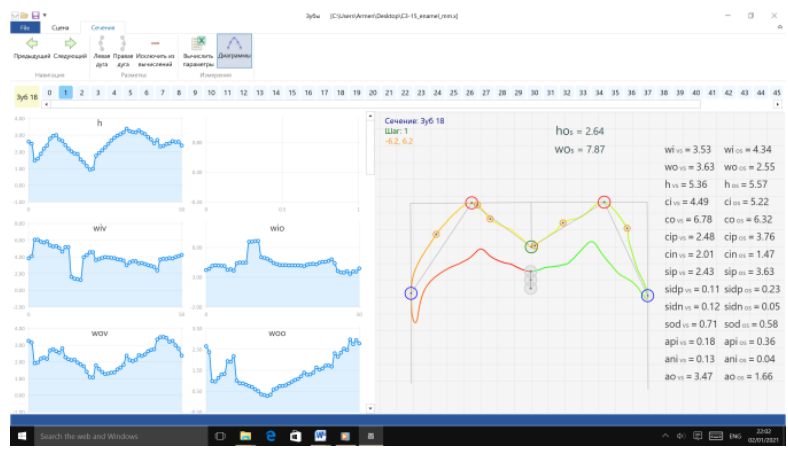

(a)

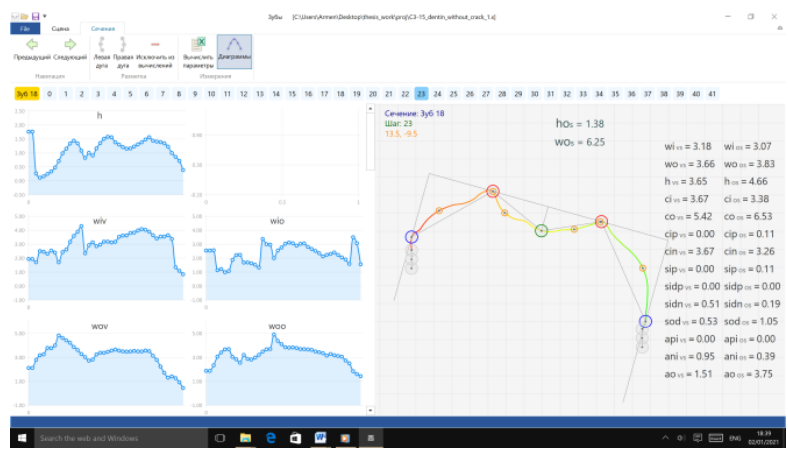

(b)

Figure 4. Measurements on enamel cap (a) and dentin (b) contours.

Some of them are measured directly, others are calculated. The complete set of parameters on every section exceeds two hundred units at this moment and this list has a potential for expansion.

\section{RESULTS AND DISCUSSION}

The algorithms of automated digital odontometry presented currently were initially developed for $3 \mathrm{~d}$ reconstructions obtained 
through optical scanning. Further development of research and wider coverage of studied issues required generation of $3 \mathrm{~d}$ reconstructions from high resolution tomographic scanning. Thus new approaches to image processing were implemented in order to match the ready-to-use algorithms to new features of reconstructions. The new requirements for images, in line with routine software functions, particularly mean generation of uninterrupted surfaces for correct running of algorithms, proper orientation and accurate measurements on enamel and dentin surfaces. The same state of affairs refers to the applied measurement techniques per se. They have been initially developed for enamel surfaces and thus their direct applicability to dentin was in question. Nevertheless the morphological interpretations forming the basis of the suggested methodology in combination with characteristics of morphology itself on various layers of dental tissues have provided the necessary conditions for conducting this study. Several upgrades allowed the method usage on the external surface of dentin.

Hence the methodology of automated digital odontometry was successfully applied to dentin external surfaces reconstructed from micro-CT scans. The entire measurement process has pauses interrupting automated algorithms. This allows the operator to verify correctness of the algorithms running interim results. For instance the stop is made when orientation and sectioning are finished. Critical evaluation can bring to necessary corrections; thus the marginal sections of dentin reconstruction were excluded from the analysis. This is due to their actual contour gave the appearance of correspondence to characteristic tooth relief although in fact would bring to misinterpretations and interfere with the results, which would show presence of a marked groove in that area (Figure 5).

Thus 40 out of 42 obtained sections were included in the study. This procedure was based on visual examination of the contour and localising its position on the dentin surface. Consequently the picked contours were excluded from count in $2 \mathrm{~d}$ section preview mode. Further measurements algorithms on dentin contours do not differ essentially from those on enamel. They are automated and supported by morphological similarities on external surfaces of both structures (enamel and dentin).

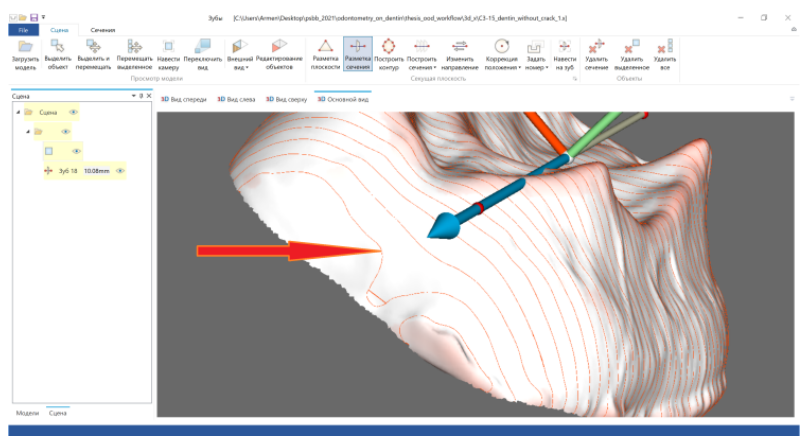

Figure 5. The red arrow points at the excluded contour.

As we have mentioned there is a wide variety of parameters obtained automatically on each contour. Nevertheless we have no intention of presenting in this paper an expanded morphological description of the studied tooth through measurements. We picked as an example the parameter of vertical dimensions of anatomical occlusal surface to present the difference of its assessment on enamel and dentin surface.

The diagram of vertical dimensions of anatomical occlusal surface parameter distribution demonstrates three expressed peaks for the dentin surface of the studied tooth. These elevations correspond to deepening of central part of the tooth (central fossa) and two elevations of outer contours on anatomical occlusal surface corresponding to the mesial and distal cusps. The values are: $1.44 \mathrm{~mm}$ on section $\mathrm{Nr} .12,1.58 \mathrm{~mm}$ on section Nr. 21 and 1.57 $\mathrm{mm}$ on section Nr. 32 (Figure 6). The arithmetic mean for the parameter on all sections accounts for $1.026 \mathrm{~mm}$.

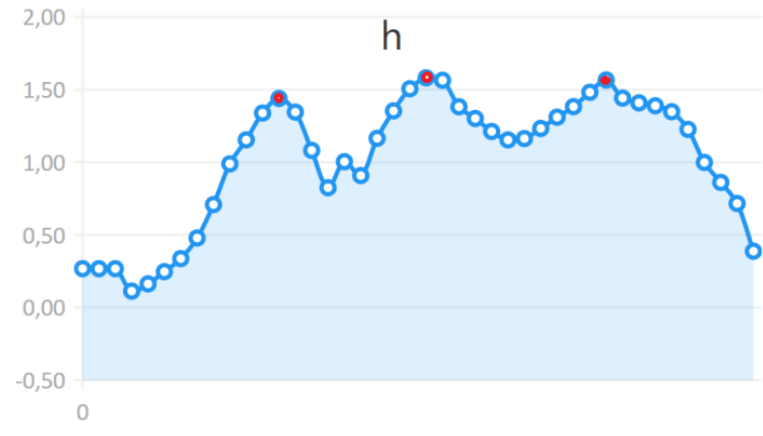

Figure 6. Red dots correspond to the peak vertical dimensions of anatomical occlusal surface on dentin.

Similar measurements were conducted on the enamel surface of the studied tooth. Here we should mention that $3 \mathrm{~d}$ reconstructions of enamel and dentin were obtained as separate models and that is why the orientation has subtle differences on each of them. In line with this there is difference in topography of these surfaces even though they have the same origin and basic principles of morphology. Nevertheless we can see on the diagram representing the parameter of vertical dimensions of anatomical occlusal surface, this time referred to enamel, very similar three peaks. They correspond to deepened areas on the enamel surface relief, which has more grooves and folds in comparison with the surface of dentin. And the parameters corresponding to maximal vertical dimension of anatomical occlusal surface on the enamel are the following: $3.42 \mathrm{~mm}$ on sections $\mathrm{Nr}$. $10,2.66 \mathrm{~mm}$ on section $\mathrm{Nr}$. 23 and $2.88 \mathrm{~mm}$ on section Nr. 33 (Figure 7). The arithmetic mean for the parameter on all sections accounts for $2.565 \mathrm{~mm}$.

Distributions of maximal results for the required parameter are different on enamel and dentin surfaces. The deepest areas are in the mesial part of the tooth on dentin surface (section $\mathrm{Nr} 32$ ) where two high elevations of mesial cusps horns are located. While the enamel surface is deeper in distal areas of the tooth (section $\mathrm{Nr} 10)$. In general, the enamel relief is more expressed and deeper than the surface of dentin. This is fully consistent with larger outer surface of the enamel cup and its masticatory function.

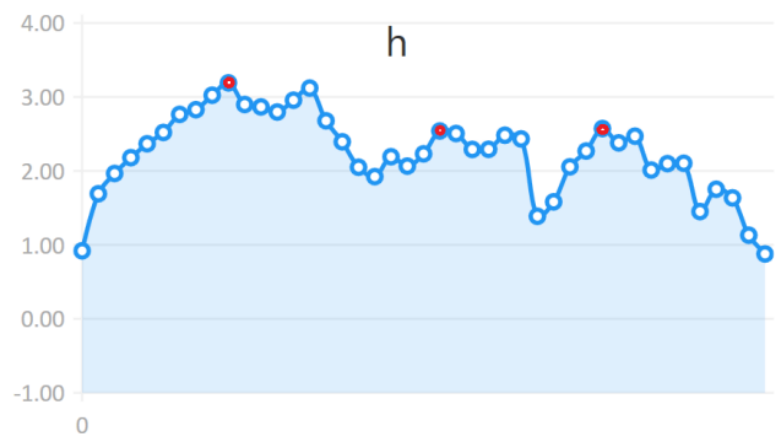

Figure 7. Red dots correspond to the peak vertical dimensions of anatomical occlusal surface on enamel. 
If we compare measurement results on enamel and dentin surfaces, we can say that our current study has revealed certain degree of correspondence of parameters obtained on morphological layers. However more profound algorithms are planned to be developed in order to avoid any discrepancy referred to different orientations of enamel and dentin reconstructions. In addition more parameters and more teeth are planned to be studied. Especially taking into consideration that list of potentially obtainable odontometric data is planned to be expanded. This tendency will be supported by micro-CT imaging as well, as it provides for more ample information on tooth coronal part and its margin which is not a distinct borderline on the majority of optically obtained images. Thus models of good practice combining imaging techniques with their processing allows revealing new data, previously unavailable in terms of quality as well as quantity neither by application of traditional odontometry, nor through visual or geometric morphometric analyses.

\section{CONCLUSION}

The suggested technique allows obtaining precise $3 \mathrm{~d}$ reconstructions of tooth enamel cap and dentin surfaces, which are applicable for automated digital odontometry. And in the current study apparently one of the first attempts to measure tooth dentin surface morphology has been presented. It has showed that digital techniques are a powerful instrument for research and their functioning principles correspond to a wide variety of studies, including dental research. This serves as an additional evidence of correctness and good potential for further development of the suggested interpretation of dental morphology.

Further improvements refer to development of fully automated algorithms for dentin measurements and more profound contour analysis including enamel margins; both directions correspond to the potential of non-invasive micro-CT imaging and reconstructive technique.

\section{REFERENCES}

Bermdez de Castro et al., 2017. Bermdez de Castro, J-M., Martinn-Torres, M., Martn-Francs, L., Martnez de Pinillos, M., Modesto Mata, M., Garcia, C., Wu, X., Xing, S., Liu, W. (2017). Early Pleistocene hominin deciduous teeth from the Homo antecessor Gran Dolina-TD6 bearing level (Sierra de Atapuerca, Spain). American journal of physical anthropology. 163. 10.1002/ajpa.23222.

Benazzi et al., 2014 a. Benazzi, S., Bailey, Sh., Peresani, M., Mannino, M., Romandini, M., Richards, M., Hublin, JJ. Middle Paleolithic and Uluzzian human remains from $\mathrm{Fu}-$ mane Cave, Italy. Journal of Human Evolution. 70. 61 - 68. 10.1016/j.jhevol.2014.03.001

Benazzi et al., 2014 b. Benazzi, S., D. Panetta, C. Fornai, M. Toussaint, G. Gruppioni, and J. Hublin (2014). Technical Note: Guidelines for the Digital Computation of 2D and 3D Enamel Thickness in Hominoid Teeth. American Journal of Physical Anthropology, 153: 305313

Gaboutchian et al., 2020. Gaboutchian, A. V., Knyaz, V. A., Novikov, M. M., Vasilyev, S. V., Leybova, N. A., Korost, D. V., Cherebylo, S. A., and Kudaev, A. A.: Automated Digital Odontometry: Measurement Data Analyses In Cases Of Complicated Dental Morphology, Int. Arch. Photogramm. Remote Sens. Spatial Inf. Sci., XLIII-B2-2020,
851856, https://doi.org/10.5194/isprs-archives-XLIII-B2-2020851-2020, 2020

Hershkovitz et al., 2018. Hershkovitz, I., Weber, G., Quam, R., Duval, M., Grn, R., Kinsley, L., Ayalon, A., Bar-Matthews, M., Valladas, H., Mercier, N., Arsuaga, J., Martinn-Torres, M., Bermdez de Castro, J-M., Fornai, C., Martn-Francs, L., Sarig, R., May, H., Krenn, V., Slon, V., Mina, W-E. (2018). The earliest modern humans outside Africa. Science. 359. 456-459. 10.1126/science.aap8369

Hublin et al., 2017. Hublin, J-J, Ben-Ncer, A., Bailey, S., Freidline, S., Neubauer, S., Skinner, M., Bergmann, I., Cabec, A., Benazzi, S., Harvati, K., Gunz, Ph. New fossils from Jebel Irhoud, Morocco and the pan-African origin of Homo sapiens. Nature. 546. 289-292. doi; 10.1038/nature22336

Krenn et al., 2019. Krenn, V., Fornai, C., Wurm, L., Bookstein, F., Haeusler, M., Weber, G. Variation of 3D outer and inner crown morphology in modern human mandibular premolars. American Journal of Physical Anthropology. Vol. 169. 05/2019, doi:10.1002/ajpa.23858

Machado et al., 2020. Machado, M., Daruge, E., Fernandes, M., Lima, I., Cericato, G., Franco, A., Paranhos, L. Effectiveness of three age estimation methods based on dental and skeletal development in a sample of young Brazilians. Archives of Oral Biology. 85. 10.1016/j.archoralbio.2017.10.014

Nadendla et al., 2016. Nadendla, L., Paramkusam, G., Pokala, A., Devulapalli, R.V. Identification of gender using radiomorphometric measurements of canine by discriminant function analysis. Indian journal of dental research : official publication of Indian Society for Dental Research. 27. 27-31. 10.4103/0970-9290.179810

Olejniczak et al., 2007. Olejniczak, A., Grine, F., Martin, L. Micro-computed tomography of primate molars: Methodological aspects of three-dimensional data collection. Vertebrate Paleobiology and Paleoanthropology. 01/2007, doi: 10.1007/978-14020-5845-5_7

Ortiz et al., 2019. Ortiz, A., Bailey, Sh., Delgado, M., Zanolli, C., Demeter, F., Bacon, A-M. A distinguishing feature of Pongo upper molars and its implications for the taxonomic identification of isolated hominid teeth from the Pleistocene of Asia. American Journal of Physical Anthropology, Vol. 170, pages 595-612. doi: 10.1002/ajpa.23928

Robinson et al., 2002. Robinson, D., Blackwell, P., Stillman, E., Brook, A. Impact of landmark reliability on the planar Procrustes analysis of tooth shape. Archives of oral biology, Vol. 47, 08/2002, pages 545-54, doi: 10.1016/S0003-9969(02)00038-9

Smith et al., 2018. Smith, T., Houssaye, A., Kullmer, O., Cabec, A., Olejniczak, A., Schrenk, F., Vos, J., Tafforeau, P. Disentangling isolated dental remains of Asian Pleistocene hominins and pongines. PLOS ONE. 13. e0204737. 10.1371/journal.pone. 0204737

Xing et al., 2014. Xing, S., Martinn-Torres, M., Castro, J., Zhang, Y., Fan, X., Zheng, L., Huang, W., Liu, W. Middle Pleistocene Hominin Teeth from Longtan Cave, Hexian, China. PloS one. 9. e114265. 10.1371/journal.pone.0114265

Yi et al., 2020. Yi, Zh., Liao, W., Zanolli, C., Wang, W. A robust alternative to assessing threedimensional relative enamel thickness for the use in taxonomic assessment. American Journal of Physical Anthropology. 174. 10.1002/ajpa.24187

Zanolli et al., 2017. Zanolli C., Bayle, P., Bondioli, L., Dean, M., Le Luyer, M., Mazurier, A., Morita, W., Macchiarelli, R. Is 
The International Archives of the Photogrammetry, Remote Sensing and Spatial Information Sciences, Volume XLIV-2/W1-2021

4 th Int. Worksh. on "Photogrammetric \& computer vision techniques for video surveillance, biometrics and biomedicine", 26-28 April 2021 , Moscow, Russia

the deciduous/permanent molar enamel thickness ratio a taxonspecific indicator in extant and extinct hominids? Comptes Rendus Palevol. 16. 10.1016/j.crpv.2017.05.002

Zubov, 2000. Zubov A.A. 2000 (in Russian) Morfologicheskoe issledovanie detey iz sungirskogo pogrebeniya 2, In: Homo Sungerensis, Verkhnepaleoliticheskiy chelovek: evolutsionnye i ekologicheskiye aspekty issledovaniya, Moscow, Nauchniy Mir, pp 256-270. 